\title{
Editorial
}

\section{Centenario de la muerte de Julio Garavito Armero}

Con ocasión del centenario de la muerte de Julio Garavito Armero (1865-1920) el próximo 11 de marzo la Academia Colombiana de Ciencias Exactas, Físicas y Naturales se vincula a las actividades programadas con este motivo publicando un número de la Revista en el cual se recoge una muestra representativa de su obra, seleccionada de entre los artículos publicados en ella desde su primer número en 1936.

En 1914 Jorge Álvarez Lleras (1885-1952) fue nombrado ingeniero ayudante del Observatorio Astronómico Nacional al lado de Julio Garavito Armero, su director, quien había sido su maestro en la Universidad Nacional. Un año después, la Sociedad Colombiana de Ingenieros solicitó a esa universidad que se estudiara la posibilidad de enviar al Congreso Científico Panamericano, programado para celebrarse en Washington en 1915, una presentación de la obra de Garavito. Álvarez y Garavito revisaron cuidadosamente la obra, particular-mente sus cuadernos, en los cuales aparecían anotaciones como "inédito", "publicado", "borrador”, etc. (Sánchez, 2007). Para esa época la salud de Garavito comenzaba a decaer y Álvarez Lleras tuvo que asumir en buena medida las labores del Observatorio, lo cual le permitió conocer muy de cerca el trabajo del Director y, sobre todo, sus ideas científicas, económicas, filosóficas y políticas. La biografía escrita por Álvarez Lleras a la muerte de Garavito fue, hasta años recientes, una importante fuente (ÁlvarezLleras, 1938) sobre la vida y obra de su maestro, hasta años recientes en que han aparecido varios documentos sobre la vida y obra de Garavito, con motivo del sesquicentenario de su nacimiento en 2015 y este año 2020 con motivo del centenario de su fallecimiento. Fue Álvarez quien acudió al Congreso Panamericano y presentó un trabajo suyo sobre meteorología, pero, aparentemente, la obra de Garavito no pudo ser presentada.

A la muerte de Garavito, Álvarez Lleras ocupó el cargo de Director del Observatorio y, unos cuantos años después, en 1936, al fundarse la Academia Colombiana de Ciencias Exactas, Físicas y Naturales, ocupó el cargo de Presidente y de director de la Revista. En esa posición Álvarez se dio a la tarea de publicar en la recién creada Revista de la Academia los trabajos de su maestro, seleccionando aquellos que a su buen saber y entender merecían divulgarse. Asumió esta tarea animado por el hecho de que todavía no se cumplían las disposiciones de la Ley 128, que rendía honores a la memoria del "sabio Profesor" (1919), entre las cuales estaba la edición de sus obras completas por parte del Estado y la erección de su busto en el jardín del Observatorio Astronómico Nacional. El busto se colocó en 1945, a los 25 años de su fallecimiento, pero la edición de sus obras completas continúa pendiente. Al respecto Álvarez Lleras afirmaba:

Poco tiempo después de desaparecido el maestro, hubimos nosotros de ocuparnos en este asunto, agitando la cuestión en prensa periódica, de acuerdo con algunos ingenieros interesados en que tal ley no se quedara escrita. Pero solo hasta ahora, veinte años después, se ha podido en este Revista dar cumplimiento a tan justa disposición, cuya realización es no solo un deber para Colombia, sino una medida atinada que habrá de traerle honra y provecho. (Álvarez-Lleras, 1939).

Después de señalar lo anterior, Álvarez Lleras citaba extensamente una nota publicada en "alguno de los periódicos de la República" en la que hacía un análisis cuidadoso de la obra de Garavito, la cual, según él, podía condensarse en dos o tres volúmenes de unas cuatrocientas a quinientas páginas y clasificarse según los diversos temas cultivados por el maestro. Se enumeran a continuación, la lista de los trabajos publicados durante la gestión de quien tanto admiró a su maestro y se preocupó porque su memoria permaneciera en la historia de la ciencia colombiana. 
Artículos de Julio Garavito Armero publicados en la Revista de la Academia de Ciencias Exactas, Físicas y Naturales entre 1936 y 1947 (los marcados con * eran inéditos).

\begin{tabular}{|c|c|}
\hline Título & Volumen (número), páginas, año \\
\hline 1. Teoría de la aberración de la luz & Vol. 1 (1): 59-65, 1936 \\
\hline 2. Nota sobre óptica matemática & Vol. 1 (2): 145-158, 1937 \\
\hline $\begin{array}{l}\text { 3. La paradoja de la óptica matemática. Teoría de la } \\
\text { aberración y de la refracción de la luz }\end{array}$ & Vol. 1 (3): 242-256, 1937 \\
\hline $\begin{array}{l}\text { 4. Óptica astronómica. Teoría de la refracción y de la } \\
\text { aberración anual }\end{array}$ & Vol. 1 (4): 334-348, 1937 \\
\hline 5. Nota sobre la dinámica de los electrones & Vol. 2 (5): 13-16, 1938 \\
\hline $\begin{array}{l}\text { 6. Una exposición elemental del método de Olbers para el } \\
\text { cálculo de una órbita cometaria * }\end{array}$ & Vol. 2 (6): 241-255, 1938 \\
\hline $\begin{array}{l}\text { 7. Principes de la Dynamique des Fluides. } \\
\text { (Principios de la dinámica de los fluidos) } *\end{array}$ & Vol. 2 (7): 366-370, 1938 \\
\hline $\begin{array}{l}\text { 8. Nota sobre las geometrías planas no euclídeas - Nota } \\
\text { sobre balística exterior }\end{array}$ & Vol. 2 (8): 566-576, 1939 \\
\hline $\begin{array}{l}\text { 9. Nota sobre la fórmula fundamental de la trigonometría } \\
\text { plana no euclídea en la geometría hiperbólica }\end{array}$ & Vol. 3 (9-10): 14-25, 1939 \\
\hline $\begin{array}{l}\text { 10. Cuestiones referentes a la astronomía - La ley } \\
\text { newtoniana es general * }\end{array}$ & Vol. 3 (11): 243-246, 1940 \\
\hline 11. El clima de Bogotá & Vol. 3 (12): 361-372, 1940 \\
\hline 12. Equilibrio de los macizos pulverulentos & Vol. 4 (13): 64-69, 1940 \\
\hline 13. Los números inconmensurables & Vol. 4 (14): 195-198, 1941 \\
\hline 14. Teoría del anemómetro de casquetes hemisféricos & Vol. 5 (17): 61-65, 1942 \\
\hline 15. Elementos de algunas funciones trascendentes enteras & Vol. 5 (17): 65-68, 1942 \\
\hline $\begin{array}{l}\text { 16. Entretenimientos matemáticos (oscilación de una barra } \\
\text { prismática sobre un cilindro recto de base circular) }\end{array}$ & Vol. 5 (19): 370-373, 1943 \\
\hline $\begin{array}{l}\text { 17. Mecánica celeste - Movimiento elíptico } \\
\text { (Método de Jacobi)* }\end{array}$ & Vol. 5 (20): 497-502, 1944 \\
\hline 18. Tablas de la Luna * & Vol. 6 (22-23): 205-213, 1945 \\
\hline $\begin{array}{l}\text { 19. Nuevos conceptos económicos: } \\
\text { (causa principal de la guerra de 1914); (evolución de la } \\
\text { distribución de la riqueza) }\end{array}$ & Vol. 6 (24): 484-490, 1946 \\
\hline $\begin{array}{l}\text { 20. Fórmulas definitivas para el cálculo del movimiento } \\
\text { de la luna por el método Hill-Brown, y con la notación } \\
\text { usada por Henry Poincaré en el tomo III de su curso de } \\
\text { Mecánica Celeste }\end{array}$ & Vol. 6 (24): 560-571,1946 \\
\hline 21. Cálculo de la efeméride del cometa de Halley & Vol. 7 (25-26): 34-46, 1946 \\
\hline $\begin{array}{l}\text { 22. Informe de la comisión observadora del eclipse total de } \\
\text { Sol del } 3 \text { de febrero de 1916, por Julio Garavito Armero } \\
\text { y Jorge Álvarez Lleras }\end{array}$ & Vol. 7 (27): 328-360, 1947 \\
\hline
\end{tabular}

De la lista anterior hemos escogido los números $5,8,9,11,18,19$ y 20 para reproducirlos en este número de conmemoración del centenario del fallecimiento de Garavito, con el fin de ofrecer una muestra significativa de su obra científica y de la diversidad de sus intereses.

Los artículos de matemática son cinco, los números 7, 8, 13, 15 y 16, todos ellos publicados en los Anales de Ingeniería entre 1897 y 1921. En Los Números inconmensurables Garavito pretende llenar los vacíos que su maestro Indalecio Liévano Reyes (18341913) había dejado en la construcción de los números reales, publicada en su Tratado 
de Aritmética de 1856. Al final, el artículo dice "continuará", pero la continuación nunca apareció. Aunque el trabajo de Liévano es digno de reconocimiento como uno de los más significativos aportes científicos en el siglo XIX (Albis y Soriano, 1977). es claro que Garavito no logró sus propósitos. En Elementos de algunas funciones trascendentes enteras el autor demuestra que estas funciones tienen algunas propiedades interesantes y el tema es tratado claramente. Se destaca la forma de encontrar el logaritmo neperiano de un entero por medio de series. Nota sobre la fórmula fundamental de la trigonometría plana no euclídea en la geometría hiperbólica es un artículo dedicado al análisis de una fórmula dada por Nicolái Lobattchewsky para la Trigonometría plana no euclídea. En él Garavito intenta demostrar que los planteamientos de este matemático, pionero en las geometrías no euclidianas, están errados por estar basados en una geometría que niega el quinto postulado de Euclides. Asimismo, en su artículo Nota sobre las geometrías planas no euclídeas, hace una fuerte crítica de estas geometrías. Dos artículos publicados posteriormente en la Revista, uno del venezolano Duarte (1946) y otro de Albis (1997), muestran con todo detalle los errores cometidos por Garavito. Lo sorprendente es que Álvarez Lleras rechazaba cualquier crítica a Garavito, como en el caso de Duarte, y, es necesario decirlo, no logró justificar, por obvias razones, las evidentes falencias en estos artículos.

En cuanto a la astronomía, sin lugar a dudas el artículo más importante de Garavito es el titulado: Fórmulas definitivas para el cálculo del movimiento de la Luna por el método de Hill-Brown, y con la notación usada por Henri Poincaré en el tomo III de su curso de mecánica celeste. Curiosamente, este artículo vio la luz al cabo de más de un cuarto de siglo de muerto Garavito. Contiene fundamentalmente desarrollos en series de potencias que son la solución de ecuaciones diferenciales que describen el movimiento de la Luna alrededor de la Tierra perturbada por el Sol con una serie de suposiciones (Luna y Sol en un mismo plano, excentricidad de la órbita terrestre nula y masa terrestre despreciable frente a la del Sol) en un sistema de coordenadas rotante. La trayectoria resultante se denomina órbita variacional. El modo de obtener las series a través de unos sucesivos e ingeniosos cambios de variable fue conseguido por primero vez por George Hill, quien hizo los desarrollos hasta la novena potencia en un pequeño parámetro. Garavito logró, en su trabajo, extender los desarrollos hasta la doceava potencia de ese parámetro. La historiografía nacional ha insistido que el propósito de Garavito era obtener unas tablas lunares y que esos desarrollos en serie eran los fundamentos para elaborar una teoría lunar "definitiva". Si ese era el propósito, los desarrollos por él obtenidos eran tan solo el primer paso, pues faltaba lo más complejo: la inclusión del movimiento del perigeo y del nodo, un esfuerzo titánico desde el punto de vista matemático. No existe ninguna evidencia de que Garavito haya emprendido el cálculo de tales desarrollos y, si pensaba hacerlo, su prematura muerte lo impidió. De modo que no es posible hablar de unas tablas lunares (las que solo es posible elaborar con una teoría lunar finalizada) con la rúbrica de Garavito. Sin embargo, Álvarez Lleras insistió numerosas veces en las páginas de la Revista sobre la existencia de tales tablas e incluso insertó unos desarrollos matemáticos en unas cuantas tablas que fundió en un artículo titulado sencillamente Tablas de la Luna y colocó el nombre de Garavito como autor. Cualquiera familiarizado con las teorías del movimiento lunar y el uso de tablas puede constatar que las de dicho artículo, por su muy reducida extensión y la omisión de una explicación para su uso, son inútiles para conseguir lo que se supone que un trabajo así debe satisfacer: obtener con gran exactitud la posición de la Luna en cualquier tiempo. Solo al final de su vida Álvarez Lleras reconoció que unas tablas lunares, en el pleno sentido de la palabra, y de cosecha de Garavito, no existían.

Por su tardía aparición, el artículo de las fórmulas definitivas no tuvo impacto alguno en el desarrollo de la teoría de Hill-Brown, utilizada en la mayor parte del siglo XX para explicar el movimiento de la Luna y, hasta donde ha sido posible indagar, solo ha sido referenciado (aparte de en las discusiones historiográficas) por el libro de enseñanza universitaria Methods of Celestial Mechanics de D. Brouwer y G. Clemence. Es muy factible que dicha citación haya pesado mucho en el Comité de Nomenclatura de la Unión Astronómica Internacional que en 1970 decidió acoger la propuesta de Jorge Arias de 
Greiff, Director del Observatorio en aquellos años, mecánico celeste consumado y, como buen ingeniero civil colombiano, admirador a ultranza de Garavito, de nombrar un cráter de la Luna con el nombre del personaje que nos ocupa.

El interés de Garavito en varios aspectos de la física se concentró en la mecánica y la óptica clásica y, en menor grado, en la mecánica de los fluidos y la termodinámica. En este campo, debe reconocerse que estaba al corriente de los avances teóricos y experimentales de su época.

Garavito asume una posición ortodoxa frente a la física, por lo que no es de extrañar que la defensa que hace de la física clásica sea acérrima. Su preocupación se orientó especialmente hacia el fenómeno de la aberración estelar y el movimiento de la Tierra con respecto al éter. Además, se ocupó del problema del crecimiento de la masa de los electrones en los tubos de Crooks, aspectos que intentó resolver desde el paradigma netamente newtoniano.

Cierto es que a finales del siglo XIX y comienzos del XX había resultados experimentales que ocasionaban serias paradojas, lo que obligaba a algunos investigadores a tratar de cambiar los aspectos fundamentales de la ciencia. Garavito juzgaba que eso era extremo. En su lugar, propuso descubrir en dónde radicaban los errores, explicarlos debidamente bajo una hipótesis novedosa, pero todo enmarcado en el contexto de la física clásica. En el caso particular del artículo Nota sobre la dinámica de los electrones, en el que señala el comportamiento observado en varios laboratorios de la relación de la carga del electrón (q) y su masa (m), la cual disminuye a medida que los electrones se acercan a la velocidad de la luz y puede conducir a la conclusión de que la masa de los electrones aumenta con la velocidad (conclusión inadmisible para algunos en aquella época), Garavito propone que, así como hay discontinuidades en la materia, bien puede suceder que las fuerzas naturales también posean discontinuidades, de modo que las "acciones eléctricas y magnéticas sean debidas a percusiones sucesivas provenientes del campo eléctrico y magnético y cuya intensidad y frecuencia determinan el valor de la fuerza". Si esto se asume, concluye Garavito, "la explicación del fenómeno es muy sencilla". Y, en efecto, con tal hipótesis y unos cálculos muy directos, logra mostrar que la desviación que sufren los electrones es proporcional a $\mathrm{q} / \mathrm{m}$. Es, por supuesto, un mecanismo ad hoc pero que constituye una muestra de la originalidad del pensamiento de Garavito en su persistente inclinación a velar por el cumplimiento de la mecánica newtoniana.

El clima de Bogotá revela otro de los intereses de Garavito, ya que, como director del Observatorio Astronómico Nacional, debía dar un informe diario al Ministro de Instrucción Pública sobre el clima de Bogotá, pero no se limitó a eso, sino que elaboró trabajos teóricos sobre meteorología (Pabón-Caicedo, 2006).

A partir de 1899, según se puede observar en sus cuadernos, Garavito comienza a preocuparse por la situación política y económica del país y a publicar algunas de sus reflexiones al respecto. Entre estos escritos, Álvarez Lleras escogió publicar Nuevos conceptos económicos: (Causa principal de la guerra de 1914); (Evolución de la distribución de la riqueza). Creemos que en la siguiente cita se resumen sus inquietudes, en buena medida válidas hoy en día.

Se ha atribuido el atraso a las instituciones políticas que han regido al país, a la religión dominante, a la raza, a la lengua, al clima, a las guerras civiles, a la falta de vías de comunicación rápidas, a la ignorancia industrial, etc. Pero, ¿cuál de estas supuestas causas se acerca más a la realidad? (Garavito, 1899)

\section{Gregorio Portilla}

Profesor Titular

Observatorio Astronómico Nacional

\section{Clara Helena Sánchez}

Profesora pensionada de la Universidad Nacional de Colombia

Miembro Correspondiente de la Academia Colombiana de Ciencias Exactas, Físicas y Naturales 


\section{Referencias}

Albis, V. Soriano Lleras, Ll. (1977). The work of Indalecio Liévano in the foundation of the real number system. Historia Mathematica. 3: 161-166.

Albis, V. (1997) Vicisitudes del postulado euclídeo en Colombia. Rev. Acad. Colomb. Cienc. Ex. Fis. Nat. 21 (80): 281-293.

Álvarez Lleras, J. (1938). Julio Garavito Armero. Estudio biográfico. Homenaje del Cabildo a la Ciudad de Bogotá en el IV centenario de su fundación. 1538-1938. Registro Municipal, Ediciones del Consejo. p. 142-154.

Álvarez Lleras, J. (1939). Notas de la Dirección. Rev. Acad. Colomb. Cienc. Ex. Fis. Nat. 3 (9-10): $1-13$.

Duarte, F. J. (1946) Sobre las geometrías no euclidianas. Notas históricas y bibliográficas. Rev. Acad. Colomb. Cienc. Ex. Fis. Nat. 7 (25-26): 63-80.

Garavito A. Julio. (1899). Causas del atraso relativo en Colombia, Cuaderno 3, 183-193, 195 $220,1899$.

Pabón Caicedo, J. D. (2006). El clima de Bogotá durante los siglos XVI-XIX a partir de material histórico. Cuadernos de Geografía Universidad Nacional. 15: 75-92.

Sánchez, C. H. (2007). Los cuadernos de Julio Garavito. Rev. Acad. Colomb. Cienc. Ex. Fis. Nat. 31 (119): 253-266. 